\title{
Congenital Hepatic Fibrosis Associated with Polycystic Kidney Disease
}

\author{
Jaimini Natvarlal Patel ${ }^{1^{*}}$, Subha Gupta ${ }^{1}$, Mansi Fauzdar ${ }^{1}$, Nisarg Patel ${ }^{2}$ and Shweta Chaturvedi ${ }^{1}$ \\ ${ }^{1}$ DNB Pathology, Santokbha Durlabhji Memorial Hospital, Jaipur, Rajasthan, India \\ ${ }^{2}$ DNB Medicine, Santokbha Durlabhji Memorial Hospital, Jaipur, Rajasthan, India \\ *Corresponding author: Jaimini Natvarlal Patel, DNB pathology, Santokbha Durlabhji Memorial Hospital, Jaipur, Rajasthan, India, Tel: 917727833722, 919909904209; \\ E-mail: jaimini1988bd@gmail.com
}

Received October 14, 2014, Accepted November 24, 2014, Published November 27, 2014

Copyright: @ 2015 Patel JN, et al. This is an open-access article distributed under the terms of the Creative Commons Attribution License, which permits unrestricted use, distribution, and reproduction in any medium, provided the original author and source are credited.

\begin{abstract}
Congenital hepatic fibrosis (CHF) is an autosomal recessive disorder and occurs as a result of ductal plate malformation. Clinically it is characterized by hepatic fibrosis, portal hypertension, and renal cystic disease. The exact incidence and prevalence of CHF are not known, but it is a rare disease. This disorder is diagnosed in most patients during childhood or young adulthood. We present the case of 8 year old female with hepatosplenomegaly, hematemesis, melena, bilateral polycystic kidney disease and a histopathological diagnosis of congenital hepatic fibrosis. She had a history of celiac disease. Congenital hepatic fibrosis belongs to the so-called fibropolycystic diseases. Celiac disease is an immune-mediated enteropathy. We describe its association with congenital hepatic fibrosis.
\end{abstract}

Keywords: Congenital Hepatic Fibrosis (CHF); Portal hypertension; Celiac disease; Polycystic kidney disease

\section{Introduction}

Congenital hepatic fibrosis (CHF) is an autosomal recessive inherited malformation defined pathologically by a variable degree of periportal fibrosis and irregularly shaped proliferating bile ducts. The hepatic manifestations of CHF were first described in 1856 [1]. The term $\mathrm{CHF}$, with its varied clinical manifestations, was recognized in 1960, and later on elaborated in 1961 [2,3]. The exact incidence and prevalence of CHF are not known, but it is a rare disease. By 1988, only 200 patients with CHF had been reported in the literature [4]. The disease appears in both sporadic (in many as 56\% cases) and familial patterns. Congenital hepatic fibrosis-ARPKD is estimated to occur in 1 in 20,000 live births [5].

Congenital hepatic fibrosis and Caroli's disease closely resemble each other pathophysiologically, in that both occur as a result of ductal plate malformation. The ductal plate is a cylindrical layer of cells that surround a branch of the portal vein, and is the embryonic precursor of the intrahepatic bile ducts, as both interlobular and intralobular bile ductules develop from the ductal plate. Progressive remodeling starts at $12^{\text {th }}$ week of gestation, and full maturation is usually complete by 20 weeks. Arrest of maturation and the lack of remodeling of the ductal plate that occurs as a result leads to the persistence of an excess number of immature embryonic duct structures. This abnormality has been termed the ductal plate malformation.

The persistence of these immature duct elements stimulates the formation of portal fibrous tissue, and it is this periportal fibrosis that contributes to the clinical picture of recurrent cholangitis or portal hypertension and associated symptoms. The ductal plate malformation has been shown to be associated with a "pollard willow" malformation of the portal vein, which results in too many small and closely branched portal veins, which supports the idea that PVCT may be congenital. Histologically, enlarged portal tracts containing immature ductal plates surrounding several hypoplastic or even obliterated portal vein branches are observed [6].

Clinically it is characterized by hepatic fibrosis, portal hypertension, and renal cystic disease [4]. The clinical manifestations of CHF are, however, nonspecific, making the diagnosis of this disorder extremely difficult. Onset of symptoms and signs is highly variable and ranges from early childhood to the $5^{\text {th }}$ or $6^{\text {th }}$ decade of life, although this disorder is diagnosed in most patients during adolescence or young adulthood [7]. The late appearance of symptoms and their clinical evolution suggest that CHF is a dynamic and progressive condition. We present it association with celiac disease.

\section{Case Report}

The 8 year old girl child was presented with abdominal distension for 2 years, history of hematemesis and melena 2 times in her life (at 6, 7 years of age). There was a history of celiac disease for 3 year. There was no history of abdominal pain or any skin bleeds and any hyperpigmentations. On tracing the pedigree, no other family member was known to be affected. She had no family history of renal cystic disease. She had a history of consangunity grade 3. General Examination revealed pallor without any sign of liver cell failure or icterus. Temperature, pulse and BP were normal. On Abdominal examination, Liver measured $8 \mathrm{~cm}$ below costal margin in epigastrium and Spleen measured $5 \mathrm{~cm}$ below costal margin with tip above umbilicus.

On Investigations, hemoglobin was $7 \mathrm{~g} / \mathrm{dl}$, Total leucocyte count was $4.6 \times 10^{3} / \mu \mathrm{l}$, Platelet count was $1.5 \mathrm{lakh} / \mathrm{mm}^{3}$. Liver function tests revealed total bilirubin was $1.8 \mathrm{mg} / \mathrm{dl}$, AST was $56 \mathrm{IU} / \mathrm{L}$, ALT was 59 IU/L and PT INR was 1.06. Ultrasound of Abdomen showed Hepatosplenomegaly with Portal Hypertensive changes, extensive periportal fibrosis with thinned out hepatic vein (Figure 1), Bilateral Polycystic Kidney Disease, Mild Ascites. USG Doppler showed normal hepatic vein. 


\section{Liver biopsy showed}

- Cores of Liver tissue showing partial loss of normal lobe of architecture of liver.

- The portal tracts are markedly expanded due to large number of anastoming biliary channels lined by cuboidal cells, some of these channels are contact with parenchymal limiting plate.

- The channels are dilated and surrounded by fibrous tissue (Figure 2).

- The liver parenchyma is nodular as the result of surrounding fibrous bands (Figure 2).

- The lumen of some of the ducts contains bile casts (Figure 3).

- The hepatocytes within nodules appear to be unremarkable (Figure 2).

- There is no inflammatory reaction in the portal tracts.

- There is no evidence of steatosis.

- Histology in consistent with Congenital Hepatic Fibrosis (Figures 1 and 4$)$.

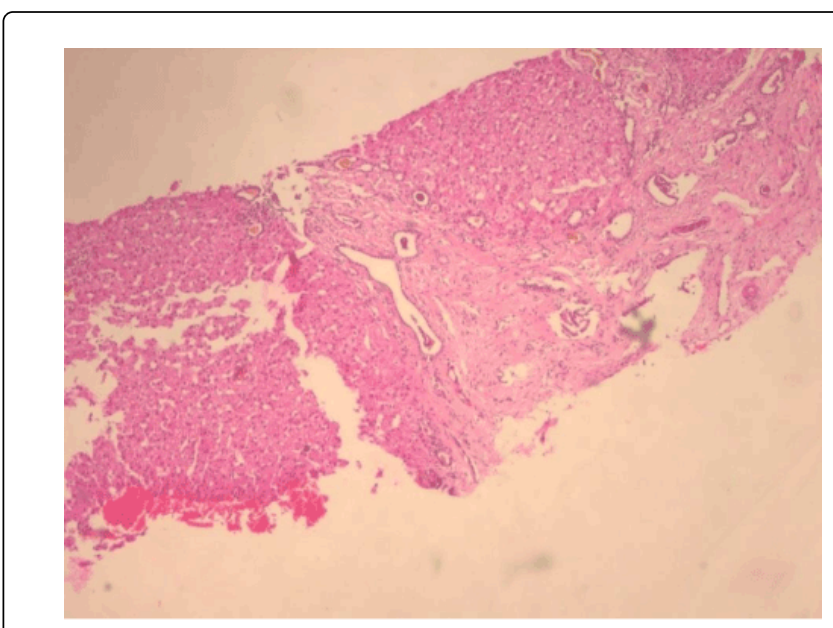

Figure 1: liver biopsy show portal fibrosis and dilated bile channels

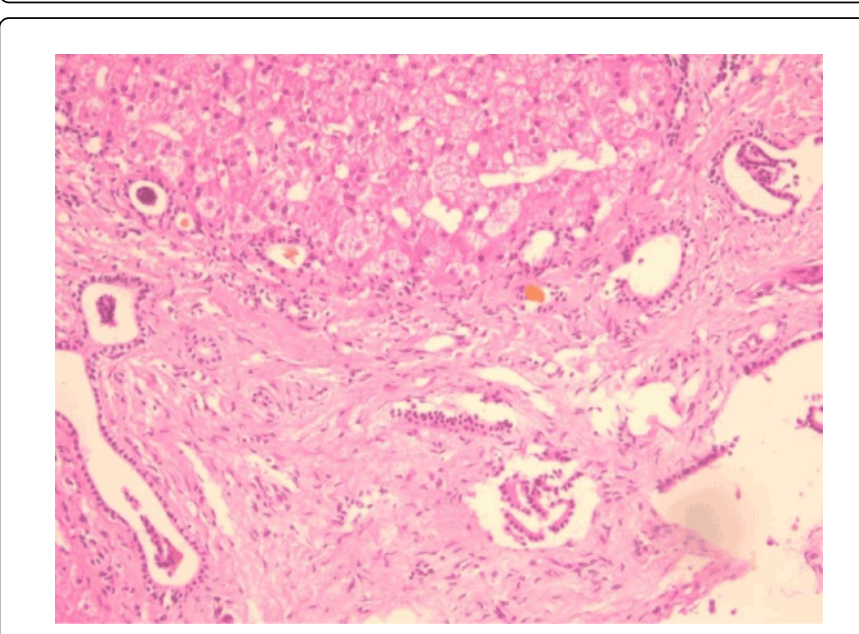

Figure 2: The channels are dilated and surrounded by fibrous tissue. The hepatocytes within nodule appear to be unremarkable

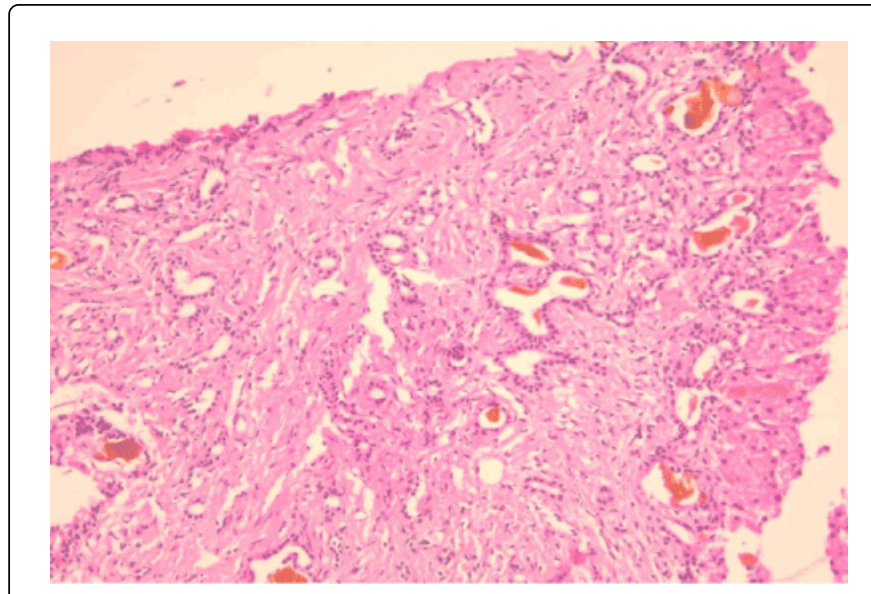

Figure 3: The lumen of some of ducts contains bile casts

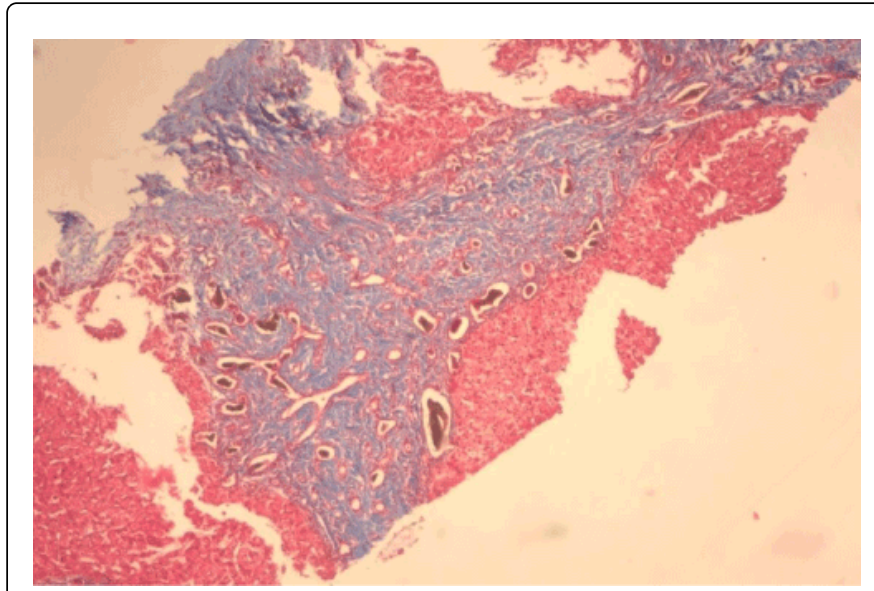

Figure 4: In Masson's trichrome stain, Liver tissue showing portal fibrosis.

\section{Discussion}

$\mathrm{CHF}$ is one of the fibropolycystic diseases, which also include Caroli disease, autosomal dominant polycystic kidney disease (ADPKD), and autosomal recessive polycystic kidney disease (ARPKD) [4].

Blyth and Ockenden have divided their patients into 4 groups called perinatal, neonatal, infantile and juvenile in accordance with the age at clinical presentation. Renal involvement is maximal in perinatal group and minimal in juvenile group [8]. Our patient had presented with hematemesis and melena (symptoms of Portal Hypertension), with no clinical or histological evidence of cholangitis and belongs to juvenile groups of this classification. CHF has initial presentation may be at around 3-6 months. The presentation ranges between 1.8-14 years, Portal Hypertension is a usual accompaniment and renal involvement is seen with $<10 \%$ tubules being affected [9]. Classically affected patients are asymptomatic until the age of 5 or 7 years when manifestations of Portal Hypertension or cholangitis lead to the diagnosis. Several clinical forms are described which depend on the variable predominance of Portal Hypertension and cholangitis. 
Cholangitis form of CHF is more severe and usually occurs in late childhood and adult life [4].

The usual presentation of $\mathrm{CHF}$ is with abdominal distension, hematemesis or melena, failure to thrive, jaundice, anemia, hepatomegaly and splenomegaly [9-11]. The other features of CHF are abdominal pain (splenic infarction), fever (cholangitis in dilated ductules), ascites, etc. [10,12-14]. CHF is particularly associated with infantile polycystic kidney disease or intrahepatic bile duct dilatation (Caroli's disease) [11]. Our case was associated with autosomal recessive polycystic kidney disease because of absence of parental history of renal cystic disease.

An unequivocal diagnosis of congenital hepatic fibrosis can only be made by an examination of a liver biopsy. The classical histological findings of this disorder are varying degrees of hepatic fibrosis with nodular formation, which may become extensive as the disease progresses. In the eyes of inexperienced pathologists, histopathological findings may easily be mistaken for cirrhosis. In CHF, widened fibrous bands may be encountered in the portal tract containing an increased number of irregularly shaped proliferating bile ducts lined by normal cuboidal epithelium. Unlike cirrhosis, hepatic lobules are usually normal with normal hepatocyte morphology, particularly in the early stages (Figure 5).

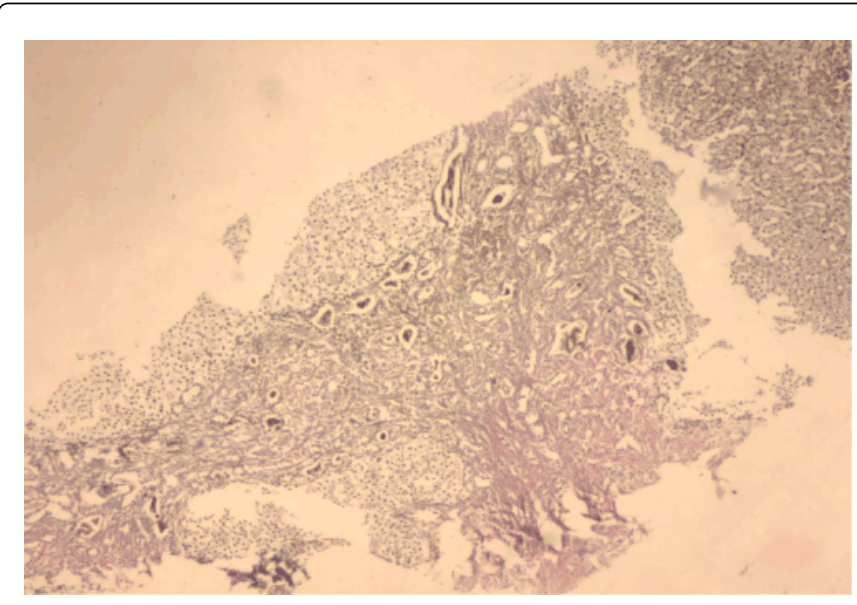

Figure 5: In Reticulin stain, liver tissue showing normal hepatocyte arrangement within nodule.

Other findings include cystic dilatation of bile ducts (Caroli's disease), and hypoplasia of the portal vein branches in association with super-numerous hepatic artery branches. In fact, congenital absence of the portal vein has been reported in a pediatric patient with CHF [15]. The cholangitis form of CHF is difficult to differentiate from Caroli's disease characterized by nonobstructive dilatation of intrahepatic bile ducts occurring as an isolated abnormality without portal fibrosis. Overlapping of $\mathrm{CHF}$ and Caroli's disease has been confirmed by histological studies [4].

Celiac disease is an immune-mediated enteropathy triggered by the ingestion of gluten containing cereals, such as wheat, rye, or barley, in genetically predisposed individuals. Celiac disease is a common disorder, with an estimated prevalence of $0.5 \%$ to $1 \%$. Biopsy specimens from the second portion of the duodenum or proximal jejunum, which are exposed to the highest concentrations of dietary gluten, are generally diagnostic in celiac disease. Pediatric celiac disease, which affects males and females equally, may present with malabsorption or atypical symptoms affecting almost any organ. Disease typically begins between ages of 6 and 24 months, after introduction of gluten to the diet, and includes irritability, abdominal distention, anorexia, chronic diarrhea, failure to thrive, weight loss, or muscle wasting [16]. Noninvasive serologic tests are generally performed prior to biopsy. The most sensitive tests are the presence of IgA antibodies to tissue transglutaminase or IgA or IgG antibodies to deamidated gliadin [17]. In a post-transplant group, $4.3 \%$ had celiac disease discovered before or after liver transplant. In this group, liver diseases recorded were primary biliary cirrhosis, autoimmune hepatitis, primary sclerosing cholangitis, and congenital hepatic fibrosis [18]. The mechanisms underlying liver injury in celiac disease are poorly defined. One probable mechanism is autoimmunity and genetic pre-disposition [19].

In conclusion, we believe that knowledge on the association between CHF and celiac disease are relevant for a better diagnosis, treatment, and follow up of patients involved.

As yet, no treatment modality has been shown to actually stop or even reverse the pathological process in congenital hepatic fibrosis, and it remains a progressive and debilitating condition. The management and prognosis of CHF is dependent on alimentary bleeding secondary to Portal Hypertension. However, prognosis may be greatly improved by shunt surgery but survival in some patients may be limited by degree of renal failure [11].

\section{References}

1. Bristowe F (1856) Cystic disease of the liver associated with a similar disease of the kidneys. Trans Pathol Soc Lond 7: 229-234.

2. Dobbs RH (1960) Congenital hepatic fibrosis with portal hypertension. Proc R Soc Med 53: 327-328.

3. Kerr DN, Harrison CV, Sherlock S, Walker RM (1961) Congenital hepatic fibrosis. Q J Med 30: 91-117.

4. De Vos M, Barbier F, Cuvelier C (1988) Congenital hepatic fibrosis. J Hepatol 6: 222-228.

5. Turkbey B, Ocak I, Daryanani K, Font-Montgomery E, Lukose L, et al. (2009) Autosomal recessive polycystic kidney disease and congenital hepatic fibrosis (ARPKD/CHF). Pediatr Radiol 39: 100-111.

6. Yonem O, Bayraktar Y (2007) Is portal vein cavernous transformation a component of congenital hepatic fibrosis? World J Gastroenterol 13: 1928-1929.

7. Zeitoun D, Brancatelli G, Colombat M, Federle MP, Valla D, et al. (2004) Congenital hepatic fibrosis: CT findings in 18 adults. Radiology 231: 109-116.

8. Blyth H, Ockenden BG (1971) Polycystic disease of kidney and liver presenting in childhood. J Med Genet 8: 257-284.

9. Summerfield JA, Nagafuchi Y, Sherlock S, Cadafalch J, Scheuer PJ (1986) Hepatobiliary fibropolycystic diseases. A clinical and histological review of 51 patients. J Hepatol 2: 141-156.

10. Abdullah AM, Nazer H, Atiyeh M, Ali MA (1991) Congenital hepatic fibrosis in Saudi Arabia. J Trop Pediatr 37: 240-243.

11. Mowat AP (1993) Congenital hepatic fibrosis. In: Liver Disorders in Childhood, 3rd edn. London, Butterworth Heinemam: 307-312.

12. Perisic VN (1995) Long-term studies on congenital hepatic fibrosis in children. Acta Paediatr 84: 695-696.

13. Ramírez Mayans JA, Mata Rivera N, Mora Tiscareño MA, Cervantes Bustamante R, Vargas Gómez MA, et al. (1994) [Congenital hepatic fibrosis. Study of 26 cases]. Acta Gastroenterol Latinoam 25: 297-303.

14. Alvarez F, Bernard O, Brunelle F, Hadchouel M, Leblanc A, et al. (1981) Congenital hepatic fibrosis in children. J Pediatr 99: 370-375. 
Citation: Patel JN, Gupta S, Fauzdar M, Patel N, Chaturvedi S (2015) Congenital Hepatic Fibrosis Associated with Polycystic Kidney Disease. J Liver 4: 171. doi:10.4172/2167-0889.1000171

Page 4 of 4

15. Gocmen R, Akhan O, Talim B (2007) Congenital absence of the portal vein associated with congenital hepatic fibrosis. Pediatr Radiol 37: 920-924.

16. Fasano A (2005) Clinical presentation of celiac disease in the pediatric population. Gastroenterology 128: S68-73.

17. Rostom A, Murray JA, Kagnoff MA (2006) American Gastroenterological Association (AGA) Institute technical review on the diagnosis and management of celiac disease. Gastroenterology 131: 1981.
18. Stevens FM, McLoughlin RM (2005) Is coeliac disease a potentially treatable cause of liver failure? Eur J Gastroenterol Hepatol 17: 1015-1017.

19. Azzam R (2010) Liver Abnormalities in celiac Disease. Impact, A publication of the University of Chicago Celiac Disease Center. 